\section{PTU-032 ALCOHOL USE DISORDERS AND LIVER FIBROSIS-CAN WE IMPROVE THE REFERRAL PATHWAY TO SECONDARY CARE?}

Freya Rhodes*, Sara Cococcia, Preya Patel, Declan Connoley, William Rosenberg. Institute for Liver and Digestive Health, UCL Division of Medicine, UCL; and The Department of Hepatology, The Royal Free London NHS Foundation Trust. , London, UK

\subsection{6/gutjnl-2019-BSGAbstracts.241}

Introduction Alcohol is the leading cause of cirrhosis in the UK, which often presents late when patients have already decompensated. Mortality from cirrhosis has increased $400 \%$ since 1970. There is therefore an urgent need for earlier detection of advanced fibrosis in primary care (PC), so that interventions can be implemented to improve outcomes. Detecting fibrosis/cirrhosis in PC is challenging as patients are often asymptomatic. There is increasing interest in the use of non-invasive tests (NIT) for liver fibrosis. We aimed to review referrals from PC to hepatology clinic for patients with alcohol use disorders (AUD), and evaluate the proportion of these patients that had evidence of advanced fibrosis.

Methods 1,657 new GP referrals to hepatology clinic at the Royal Free Hospital from Jan 2015-Jan 2017 were reviewed, and those with suspected alcohol-related liver disease (ALD) as reason for referral were selected and analysed. Data were collated on demographics, fibrosis staging, reason for referral and alcohol use.

Results 141 patients were referred with suspected ALD (71\% male, median alcohol intake 70 units/week) (IQR 49-140). Most patients $(64 \%, 90 / 141)$ were referred on the basis of abnormal liver function tests (LFTs), alcohol history, steatosis on ultrasound (US), or examination findings. Of those referred $24.8 \%(35 / 141)$ had US findings of chronic liver disease prior to referral. Of these, $34 \%(12 / 35)$ were subsequently deemed not to have advanced fibrosis or cirrhosis in secondary care. Prior to referral, $89 \%(125 / 141)$ of patients had not had a NIT for liver fibrosis. Once seen by hepatology, only $36.2 \%$ of the referred patients (51/141) were confirmed to have either advanced fibrosis or cirrhosis (by fibroscan, imaging, biopsy or ELF), and were kept under follow up. The remaining $63.8 \%$ were discharged back to PC and represent unnecessary referrals that may have been avoided through the use of NIT in primary care. Current BSG guidance does not recommend routine NIT in patients with AUD drinking <35/50 units/week(F/M) with AUDIT score $<19$ but in this review of patients referred from PC a diagnosis of advanced fibrosis or cirrhosis was confirmed in $3 / 23$ of male patients drinking 14 49 units/week.

Conclusions We propose that the use of NIT in PC patients with AUD would significantly reduce the number of 'unnecessary' referrals to secondary care, and increase the earlier detection of advanced fibrosis. US cannot always be relied on for a diagnosis of cirrhosis. Further research is needed to determine which thresholds of alcohol intake warrant application of NIT, and it would be interesting to repeat this study in 2020 to evaluate the impact of the 2017 BSG LFT guidelines.

\section{PTU-033 HEPATITIS C; PATHWAYS REACHING THE POPULATION OTHER PATHWAYS CAN'T REACH}

${ }^{1}$ Emma Robinson*, 'Paul Brennan, ${ }^{2}$ Francesco Manca, ${ }^{2}$ Kathleen Boyd, ${ }^{1} J o h n$ Dillon. ${ }^{1}$ University of Dundee, Dundee, UK; ${ }^{2}$ University of Glasgow, Glasgow, UK

\subsection{6/gutjnl-2019-BSGAbstracts.242}

Introduction NHS Tayside is committed to eradicating the Hepatitis C virus (HCV) by 2030 in line with the World Health Organisation recommendations. This includes diagnosing at least $90 \%$ of those infected. With an estimated prevalence of $0.55 \%$ we anticipate that we should have 2700 people with chronic HCV. 2300 are diagnosed and have either been treated and cured or are in line to be treated. We anticipate that a further 400 are un-diagnosed. It is vital that we diagnose and treat these individuals in order to prevent further transmission of the virus and achieve elimination.

NHS Tayside have instituted a number of specialised pathways for testing and treatment of HCV amongst the most atrisk populations, including people who inject drugs (PWID), those on opiate substitution therapy and prison inmates. Widespread testing occurs in injecting equipment provision sites (IEPS), community pharmacies, substance misuse centres and the prison service.

Our aim was to analyse the efficacy of these directed diagnosis pathways compared with standard testing.

Methods Data was collected for every Hepatitis C IgG and PCR test ever done in NHS Tayside. We attributed each test to a diagnosis pathway according to the testing source. Clinical records show testing source for every individual in NHS Tayside with positive antibody results. Pooling this data allowed us to assess pathway efficacy.

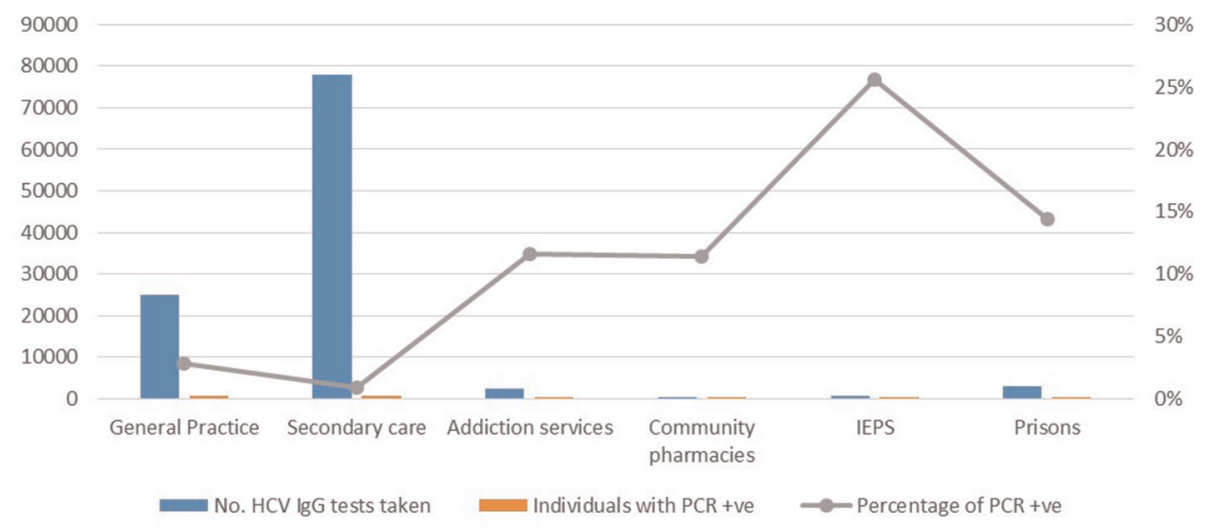

Abstract PTU-033 Figure 1 Rate of HCV PCR positive tests by testing site 
Results Overall the diagnostic activity for HCV has increased over the last two decades. More markedly since 2012 when DAAs were becoming available. The standard diagnostic pathways (primary and secondary care) show large volume testing with a low rate of PCR positivity. In contrast testing pathways aimed at high risk individuals show a higher PCR positive rate. See figure.

Conclusions Utilisation of diagnostic pathways targeting populations most at risk of $\mathrm{HCV}$ are more effective at yielding new HCV diagnoses than standard pathways. These tailored diagnostic pathways will also resolve some of the health inequalities around drug use and provide methods of ensuring entry to treatment. We believe using targeted testing will find the majority of our undiagnosed population. This will help us to direct resources and achieve our aim of elimination by 2030.

\section{PTU-034 SPLEEN STIFFNESS BY ELASTPQ POINT SHEAR WAVE ELASTOGRAPHY PREDICTS CLINICALLY SIGNIFICANT PORTAL HYPERTENSION IN PBC}

\footnotetext{
1,2Francesca Saffioti" ${ }^{*}$ 'Davide Roccarina, ${ }^{1}$ Matteo Rosselli, ${ }^{1,3}$ Roberta Stupia, ${ }^{1}$ Aileen Marshall, ${ }^{1}$ Massimo Pinzani, ${ }^{1}$ Douglas Thorburn. ${ }^{\prime}$ Sheila Sherlock Liver Centre, Royal Free London NHS Foundation Trust and UCL Institute for Liver and Digestive Health, University College of London, London, UK; ${ }^{2}$ Department of Clinical and Experimental Medicine, University of Messina, Messina, Italy; ${ }^{3}$ Division of Clinical and Molecular Hepatology, University Hospital of Messina, Messina, Italy
}

\subsection{6/gutjnl-2019-BSGAbstracts.243}

Introduction There is an ongoing effort to identify non-invasive surrogates for staging liver disease and detecting clinically significant portal hypertension (CSPH), defined as the presence of gastro-oesophageal varices on endoscopy. The correlation of spleen stiffness measurement (SSM) with portal hypertension measured by hepatic venous pressure gradient has been reported, but data regarding its reliability are controversial.

We assessed the ability of liver stiffness measurement (LSM) and SSM performed by point-shear wave elastography (pSWE) to detect $\mathrm{CSPH}$ in primary biliary cholangitis (PBC).

Methods Predictors of CSPH were evaluated in $53 \mathrm{PBC}$ patients who had an upper-GI endoscopy within 12 months of the elastographic assessment. Demographics, biochemical and ultrasonographic data were prospectively collected. Transient elastography (Fibroscan, Echosens) [F-TE], liver and spleen pSWE (ElastPQ, Philips Affiniti70G), and the most common clinical fibrosis scores were obtained. ROC curves were constructed to establish the performance of elastographic techniques and fibrosis scores in predicting CSPH.

Results Mean age $57 \pm 12$ years, 91\% female. OVs were detected in 12 (23\%) cases (4 at high-risk of bleeding).

Variables significantly associated with CSPH at univariate analysis were: diagnosis of cirrhosis, Child-Pugh, MELD and Mayo risk score, spleen area and longitudinal diameter, platelets, albumin, ALT, INR, F-TE, ElastPQ LSM and SSM, LSPS (LSM*spleen diameter/platelets). SSM remained the only independent predictor of CSPH in all the multivariate models (OR 1.101; 95\%CI 1.14-1.20; $\mathrm{p}<0.022$ ).

F-TE and ElastPQ LSM showed good AUROCs (95\%CI): $0.85(0.72-0.99)$ and $0.80(0.62-0.98)$, with best cut-off 14.3 and $13.8 \mathrm{kPa}$, respectively. However, specificity $(\mathrm{Sp})$ was $<70 \%$ for both tests. ElastPQ SSM showed the best performance in predicting CSPH (AUROC 0.95, 95\%CI 0.87-1.00, sensitivity (Se) $88 \%$, Sp 97\%), with optimal cut-off $50.1 \mathrm{kPa}$
(PPV 0.89, NPV 0.97), followed by GUCI, King's score and LSPS [AUROC (95\%CI): 0.94 (0.87-1.00), 0.94 (0.85-1.00), 0.93 (0.85-1.00), respectively] (fig. 1).

In this cohort, the diagnostic performance of ElastPQ SSM in detecting CSPH was superior to the recently validated Baveno VI and Expanded Baveno VI criteria, which showed good Sp (77\% and 87\%, respectively) but low Se $(67 \%$, for both).

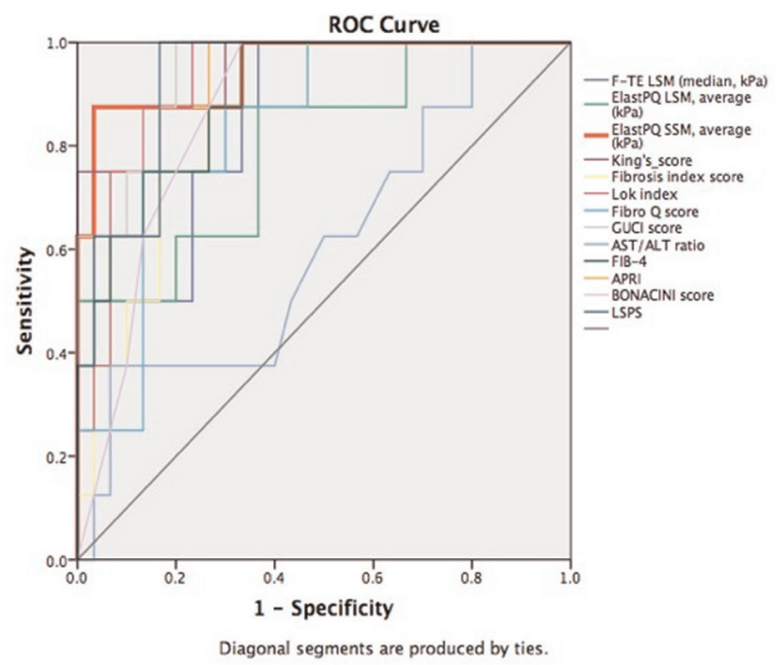

Abstract PTU-034 Figure 1

Conclusions We provide evidence that transient elastography, in particular ElastPQ SSM, can be used as a reliable tool for the detection of CSPH in PBC.

\section{PTU-035 ECONOMIC IMPACT OF NON-RESPONSE TO URSODEOXYCHOLIC ACID IN PRIMARY BILIARY CHOLANGITIS PATIENTS}

${ }^{1}$ Parivrudh Sharma*, 2 Lynne Crighton, ${ }^{3}$ Balasubramaniam Vijayan, ${ }^{3}$ Shirley English, ${ }^{3}$ Ashis Mukhopadhya. 'University of Aberdeen, Aberdeen; ${ }^{2}$ Pharmacy Department, Aberdeen Royal Infirmary, Aberdeen; ${ }^{3}$, Department of Digestive Disorders, Aberdeen Royal Infirmary, Aberdeen

\subsection{6/gutjnl-2019-BSGAbstracts.244}

Introduction NICE recommends the use of Obeticholic acid (OCA), as a second-line treatment for failed or intolerant ursodeoxycholic acid (UDCA) drug therapy in primary biliary cholangitis (PBC). This audit aims to determine the proportion of PBC patients, in a tertiary referral hospital experiencing failed UDCA drug therapy, to gauge the potential economic impact of a switch to OCA.

Methods A total of 120 patients with PBC were identified from an existing patient database. 24 patients were excluded due to inappropriate diagnosis, missing data, non-attendance or failure to tolerate UDCA. Baseline characteristics, UDCA dosage and biochemical response were recorded for all patients. For the purpose of this study, failed UDCA drug therapy was defined as an alkaline phosphatase (ALP) level of greater than 1.67 times the upper limit of normal (ULN) (Toronto Criteria) ${ }^{1}$.

Results Of the 96 patients included for analysis, 9 were male and the remaining 87 were female. The mean age and weight 